\title{
Shaping the Future with Flexible, Wire-Shaped Supercapacitors
}

\author{
Joe Sleppy ${ }^{1}$, Dr. Isaiah Oladeji ${ }^{2}$ \\ ${ }^{1}$ Capacitech Energy, Inc. \\ 3259 Progress Drive, Orlando, FL 32826 \\ joe.sleppy@capacitechenergy.com; i.oladeji@capacitechenergy.com
}

\section{Extended Abstract}

Supercapacitors are critical to the mainstream adoption of a variety of technologies enabling Cleantech, Wearables, and Internet of Things (IoT) applications. While the value added by supercapacitors is known and well documented [1], finding the physical space to incorporate supercapacitors in those systems is another challenge to overcome.

Supercapacitors are physically rigid energy storage components that come in a cylindrical or rectangular shape that are used on printed circuit boards (PCBs), where space is highly valuable. This traditional shape limits an engineer's placement options in terms of where to use supercapacitors, making it difficult to use them in certain applications. This restriction forces engineers to make difficult design and performance sacrifices.

Capacitech Energy, Inc. is commercializing research from the University of Central Florida that reimagined the shape of supercapacitors to be physically flexible and wire-shaped [2]. The innovation leverages its novel form factor to eliminate the need to make painful engineering trade-offs. This is because, like no supercapacitor before it, it can be routed through areas where space is available on the PCB or placed off the PCB and integrated into other parts of the product or system's infrastructure instead, such as a wiring harness for an electric vehicle. While offering unparalleled design flexibility and alternative placement options to integrate supercapacitors into the world's infrastructure, the new shape also leverages a geometric advantage to increase its capacitance by $\sim 20 \%$ compared to the same electrodes built in a parallel plate form factor. These key features and its high-power density make this technology well suited to complement energy harvesting technologies and batteries used in a variety of applications.

One attractive use case for this wire-like supercapacitor is in the IoT space. Limited by a low cycle life, battery powered IoT devices have a short service life. The cost of frequently replacing these batteries every 2-5 years is staggering. A technician can expect to spend $20 \%$ of their day changing batteries for a typical IoT installation. Energy harvesting technologies, such as a solar cell, on their own are extremely limited due to their low peak-power capability; however, they can be paired with a high-powered supercapacitor to serve as an alternative to batteries. This technique is growing in popularity, but also forces design substitutions to free up space on the IoT device's PCB to make room for supercapacitors. Instead of using space on the PCB, the cable form factor can be connected with the wires coming from the energy harvesting device [3]. Capacitech's supercapacitor features 3F of capacitance, can be charged at low currents, and is capable of providing over 1A of current to the load [4]. The result meets the power requirements of Bluetooth, Wi-Fi, LoRa, and even cellularly connected IoT devices. Economically, it could reduce the cost of ownership for the end user (by replacing costly batteries) by $\sim 25 \%$ and returns $\sim 33 \%$ of the space on the IoT device's PCB to the design engineer for miniaturization or new features.

A similar approach can be used to overcome the trade-offs from using supercapacitors as part of the energy storage system in a variety of applications. Many opportunities exist to complement batteries with this cable-based supercapacitor's peak-power capability and to build cable-based backup power systems. This innovation helps designers meet their goals by building a discrete and distributed network of supercapacitors, made possible by this innovative form factor. This innovation is what engineers need to build the next generation SmartCity electronics, wearables, solar power systems, electric vehicles and more.

\section{References}

[1] None. (2020, December 08). BU-209: How does a Supercapacitor Work? [Online]. Available: https://batteryuniversity.com/learn/article/whats_the_role_of_the_supercapacitor

[2] Z. Yu and J. Thomas, "Coaxial supercapacitor-electrical cables: Integrating energy storage device into electrical cables," Adv. Mater. 26, 4279 (2014). 
[3] J. Sleppy. (2021, March 5). Flexible Supercapacitors for Energy Harvesting Technologies in IoT [Online]. Available: https://www.capacitechenergy.com/blog/flexible-supercapacitors-for-energy-harvesting-technologies-in-iot

[4] Mouser Electronics, Inc. (2021, March 19). Cable-Based Capacitor [Online]. Available:

https://www.mouser.com/new/capacitech/capacitech-cable-based-capacitor/ 